Session 2553

\title{
Making Clocks: A First-Year Course Integrating Professional Communications with an Introduction to Engineering
}

\author{
W. Bernard Carlson and Karin Peterson \\ University of Virginia
}

As engineering educators worry about attracting and training outstanding undergraduates, it has become important to develop strong first-year courses that introduce students to engineering as a discipline and a profession. A strong introductory course is vital because first-year engineering students arrive at the university with a variety of needs which must be addressed immediately if they are to have four productive years. Fresh out of high school, students often come to engineering school with only a hazy notion of what engineers do and what kind of knowledge engineers use. As students take up the standard requirements in calculus, chemistry, graphics, computer languages and professional communications, they frequently need to have an exciting and meaningful learning experience, a creative experience that motivates them to stay in engineering. To survive the heavy math and science load, students must also acquire new study skills; in particular, to master new abstract concepts, they need to learn how integrate the words, diagrams, and equations used to explain those concepts in their textbooks. In their communications class, first-year students are often frustrated to discover that the expository and interpretive writing skills learned in high school do not necessarily prepare them to write in a precise, analytical, and professional manner. But above all, first-year students need to acquire a framework for thinking about engineering, a framework which will help them to integrate the ideas and skills which they will acquire during their four-year education.

To meet these many needs of first-year students, engineering schools have developed a variety of introductory courses. Frequently, these courses introduce students to either the different branches of engineering or some of the tools of engineering--basic concepts, mathematical techniques, and software packages. At a few schools, the introductory course is now a design class in which the students are asked to come up with a creative solution to a problem as a way of whetting their appetite for the more demanding courses to come. ${ }^{1}$ At the University of Virginia, first-year students currently take three entry-level courses. Engineering 160 covers basic software packages (Mathcad, Silverscreen, and Quattro Pro) and Engineering 164 introduces design.

While these two courses provide UVA frost-year students with many of the tools and concepts they need for their engineering studies, a third course provides an intellectual introduction to engineering. In our sections of TCC 101, Language Communications in a Technological Society, we challenge the students to think about the nature of engineering by having them build a mechanical clock timer kit and then preparing a series of writing and speaking assignments related to the clock. The writing assignments include maintaining an engineering notebook, revising the kit's assembly instructions, and preparing an illustrated technical description. The students also work in teams to design an improved clock timer, draft a patent application, and prepare a proposal for manufacturing and marketing their improved versions. To hone their public speaking skills, the student teams are required to present their patents and manufacturing proposals before the class. By combining this hands-on exercise with communications assignments, this course helps students to appreciate the range of activities (design, analysis, patenting, and cost calculations) that constitute engineering, and it prepares them to understand and appreciate the variety of courses they will take over the remainder of their undergraduate education. 


\section{Course Assumptions}

On one level, this course may seem to be simply a sequence of technical writing assignments, the novelty of which is that the students actually build the device about which they are writing. On another level, however, we have selected and structured the assignments on the basis of three key assumptions about the nature of engineering, human cognition, and the pedagogy of writing.

The first assumption concerns the nature of engineering. It is our belief that engineering fundamentally involves controlling the forces of Nature and working with physical materials. An engineer may be far removed intellectually and physically from the actual forces involved in his or her designs, but what makes engineering a distinctive profession is that the calculations, models, and simulations will sooner or later be used by someone to build an actual device. An engineer's calculations or computer modelling may seem highly abstract and removed from reality, but this work only counts if it can be connected to the physical world. Because students spend much of their time studying abstract ways for analyzing the forces of Nature, it is becomes highly important for them to have an exercise early on in their education where they actually build a real device, particularly one which harnesses simple forces (The clock timer kit uses a pendulum and a falling weight). In this way, building the clock timer kit is a powerful lesson, reminding the students that engineering is about building real objects which need to work reliably in the real world.

A second assumption concerns how humans learn about the material world and gain mastery over it. Some cognitive scientists would argue that we solve problems by creating different kinds of representations. ${ }^{2}$ By picturing a problem using different images, words, symbols, or numbers, humans are able to isolate key factors, identify patterns, and come up with a solution. Frequently, we use a range of different representation techniques-we take notes, make sketches, write equations--to acquire and organize information about the world. In engineering education, we pride ourselves on teaching students a host of representational techniques--complex mathematics, powerful computer models, precise ways of drawing, and rigorous ways of writing and speaking-and we teach these techniques so that students can analyze, predict, and eventually control the forces of Nature. While we as engineering educators and practitioners intuitively know that the power of our profession is grounded in our ability to represent the natural world, we frequently fail to convey this essential lesson to our students. ${ }^{3}$ In our course, we seek to call attention to the central role that representations play in technological problem solving, and we do so in order that students may use this idea to structure their future studies.

A third assumption concerns how one teaches students to write more effectively. Composition teachers have found that students not only need lots of practice writing but they also need the opportunities to apply what they learned on one assignment to another. It is important for students to have assignments which build upon one another, that allow them to utilize the instructor's comments on the next paper. Consequently, rather than having the students do a series of writing exercises that are only tangentially related, we have designed this course around a sequence of assignments which build upon one another. For example, after a writing a technical description of how the clock timer kit works, we explain to the students that their patent applications should include a technical description of how their improved versions works, and that where appropriate, they should use revised portions of the previous assignment. By not only permitting but encouraging them to "recycle" old assignments, we teach students valuable skills in editing and revising.

\section{Building the Clock Timer Kit}

Let us turn now from the assumptions underlying the course to describing the various activities and assignments. [For an overview, please see the schedule grid in the Appendix]. The first order of business is, quite naturally, building the clock, and we give the students three weeks outside of class to construct and troubleshoot the kit.

The students purchase a clock timer kit manufactured by Woodkrafter Kits of Yarmouth, Maine. Woodkrafter Kits is a medium-size toy manufacturing company, and the University Bookstore finds it relatively easy to order these kits and stock them on the shelves alongside the textbooks. Woodkrafter Kits offers an educational discount, so that even with the bookstore markup, the kits cost the students under $\$ 6.00$. 
We initially chose this clock timer kit because it is made up of wooden and cardboard parts and requires no special tools for assembly. Since the kit is intended for children ages 10 and up, we assumed that first-year engineering students would find it easy to assemble this kit (but more on this assumption in a moment).

However, once the students started building the kits, we discovered several additional features that made the kits a useful learning tool. First, much to the students' chagrin, the kits are often missing parts, and students are challenged to fashion replacement parts out of scrap material in the kit or whatever they can find in their dorm rooms. Second, the students quickly discover that this is not a precision kit--the cardboard parts are often poorly die-cut, and the students need to trim and work the materials quite carefully. These first two features may seem unfair and annoying, but we find the problems with the parts to be a perfect opportunity for driving home lessons about precision and quality in manufacture.

While each student is expected to assemble a clock and get it to run by fifth week of the semester, we do provide several forms of support. We set aside one class period in which the students can work on their clocks, and they often learn a great deal by comparing their clocks-in-progress. We also provide an assembled clock for them to examine. To help the students track their problems with the kit and the instructions, we discuss how to keep an engineering notebook in which students are expected to make observations and sketches. ${ }^{4}$ Finally, during these early weeks, the TA set aside special office hours for answering questions about the kits.

Despite these forms of support, many students find it exceedingly difficult to get their clock timer kits to actually run. On the due date for the clocks, only about 20 percent of the students are able to demonstrate a clock in which the pendulum consistently regulates the descent of the weight. While we initially worried that female students would have difficulty in getting the clock to operate since they presumably lacked previous experience with models and mechanical devices, we have found that female students had no such general difficulty, and the females did as well their male counterparts.

In assembling the clock, students have a hard time anticipating how the various parts should fit, particularly how the gears should mesh. They also fail to appreciate the importance of having the shafts and gears parallel or perpendicular to each other. We assumed that students would generally know that a key part such as the lantern gear needs to have its dowel teeth at right angles from the edge in order to mesh with the power gear, but students consistently assemble the lantern gear with crooked teeth since that is how the parts "seem" to fit together.

Once the clock is assembled, the students also find it difficult to observe and diagnose problems. They seem to lack simple trouble-shooting heuristics such as tracing the force exerted by the weight through the gear train and looking for points where the force is blocked. To help with trouble-shooting, we have students work in groups in class, with several frustrated students teaming up with a student whose clock works. However, in future versions of the course, we will spend more time explicitly introducing students to trouble-shooting strategies.

\section{Overview of the Writing and Speaking Assignments}

Revising the Instructions. Once the students have more or less gotten their clocks to run, we turn to the first major writing assignment, revising the assembly instructions. The kit comes with a 12-page booklet which describes step-by-step how to assemble the clock. It is illustrated by a number of detail sketches and includes one overall diagram of the assembled clock. Students quickly discover and complain that these instructions violate a whole range of technical writing rules--information about how the parts are supposed to fit together is missing, warnings are given at the end of a step, not at the beginning, and the illustrations fail to convey the exact relationship between parts. Capitalizing on their frustration with the instructions, it is easy to get the students to take up the task of rewriting a portion of the instructions. To do so, they are asked to review their notebooks for problems they noted in the course of assembly. In class, we review general guidelines for writing instructions using Anne Eisenberg's Effective Technical Communications. 5 Since the students are required to not only change the words but also the pictures in the instructions, we discuss what makes for effective diagrams by using selected chapters from Donald Norman's Things that Make Us Smarter 2 Since students are just learning a graphics package (Silverscreen) in ENGR 160, we do not require the students to prepare formal diagrams but 
permit them to submit hard-drawn sketches which convey the sort of diagram they think is needed at various points in the instructions.

The Technical Description. As a second exercise, we ask the students to write a 2-3 page illustrated explanation of how the clock timer works. In preparation for this assignment, the students read Thomas Edison's patent for the phonograph.6 This brief patent serves as a model of effective technical description since it reveals how the sequence of a definition, discussion of form, and description of function can convey the essential elements of a device. We also examine how Edison linked the patent drawings to the specification through the use of labelled parts. Finally, we use Edison's patent to discuss how engineers must be able to integrate words and pictures in their minds in order to understand a device, theory, or mathematical concept.

As students complete their descriptions, we lecture on the invention of clocks in late Medieval Europe and on how contemporary engineers can analyze the clock timer mechanism in terms of forces and torques. ${ }^{7,8} \mathrm{We}$ cover the second lecture topic not because we expect first-year students to be able to perform this sort of dynamic analysis but rather to help them link the clock project to what they are learning in their engineering courses. Students find it quite stimulating at this point to reflect on how Medieval craftsmen were able to invent clock mechanisms and to compare a craft understanding of clocks with the mathematical analysis of modern engineers. Having struggled to build their own clock, the students become quite interested in how inventors and engineers solved problems relating to clocks, and thus the lectures problematize the issue of problem-solving for the students. Confronted with several ways to "know" about clocks, students no longer take it for granted that there must be one way to think about and solve engineering problems.

Improving the Clock Timer: The Patent Assignment. As the students become confident in their understanding of how the clock timer works, we challenge them to improve upon the design and to prepare a patent documenting their improvements. From a writing standpoint, the patent assignment is a logical next step since it combines a technical description similar to what the students have just written with the new element of writing claims which precisely delineate what is new about their design. While the instruction and technical description assignments were prepared by students working alone, the patent exercise is a group effort, and thus gives the students an opportunity to begin developing teamwork skills.

To simulate the real-world process of patenting, the students are required to review patents developed by students in previous years. Students study the earlier patents because they are required to come up with an improvement that does not infringe on existing patents. Since students know little about patents and why inventors and engineers try to secure them, we devote several lectures to the history of U.S. Patent Office and to writing claims. As a source for claim writing, we use David Pressman's Patent it Yourself! 9

To complete the simulation, the student groups are required to present their patents to the class and two outside patent "examiners." Unlike public-speaking exercises in other classes where the teacher and audience are polite and passive, the examiners are free to interrupt the student presentations, ask questions, and challenge assumptions. The examiners are especially hard on students who claim that their presentation or specification doesn't quite capture what they designed; the examiners remind the students that they can only get a patent for what they can communicate. At the end of each group's presentation, the examiners rule on which claims can be accepted and which are rejected. Needless to say, students find the examination process scary and unpleasant, but it does make them realize they need to choose carefully the words and diagrams they use to describe their improvements. To date, the examiners have simply been graduate students who are familiar with the exercise but not necessarily patent law. In the future, we would like to invite engineers or patent lawyers to the class to play the part of patent examiner, in the hope that they would help make the simulation even more realistic.

The Manufacturing Proposal. Now that the student groups have developed a new product, the final assignment is to explore what it would take to produce and sell their designs. To do this, each group prepares a ten-page proposal which outlines a plan for manufacturing and marketing their improved clock timer. In this proposal, the students assume they are asking a bank or group of venture capitalists for a specific amount of money to support the first year of their new business. Hence, the students must think carefully about what sort of information these investors need to know, and they need to present this information in a highly persuasive manner. 
To simplify the process, the students are given several basic assumptions. We instruct them to plan on selling the clock only in Charlottesville and the surrounding area which has a population of approximately 100,000. The students are also told to assume that there are no competitors, no taxes, and no regulations. Finally, the groups are advised that they are not required to show a return on the bank's investment, but they are reminded that some profit to the investors would enhance their chances of getting the loan.

To prepare their proposals, the groups undertake a substantial amount of planning and thinking. They must consult 1990 Federal census data to estimate the size of their potential customer groups, develop a scheme for assembling the clocks, decide on what parts or tasks will be outsourced, identify a marketing channel, determine the costs of manufacturing and marketing, and calculate a suitable retail price. In designing their proposals, the students consult a chapter on proposals in Eisenberg, ${ }^{5}$ but the groups are also advised to think carefully about the level of detail and the sequencing of information in order to make the proposals persuasive. And we frequently remind the students that they are trying to get investors to loan them potentially tensor hundreds of thousands of dollars!

The ten-page proposals generally include a description of the product, the target market, and the manufacturing process The students are required to provide diagrams showing the product and manufacturing process as well as appropriate tables and graphs displaying costs. To prepare these drawings and tables, the students automatically turn to using the Silverscreen and Quattro Pro which they learn in ENGR 160. As with the patent, each student group is required to present their manufacturing proposal to the class, with each member required to speak.

Most students genuinely enjoy the manufacturing proposal assignment because it allows them to utilize much of what they have learned during the course about clocks, writing, and design. The groups often become quite excited about developing imaginative but profitable schemes for manufacturing and marketing schemes for their clock products. This past year, one group designed a grandfather clock which they expected to sell as a piece of fine furniture while another group proposed manufacturing a game timer which they would sell to the Milton Bradley Company. Frequently, the students exhibit their pride in their products by creating a name and logo for their company, neither of which is required.

\section{How Well Does the Course Work?}

In a questionnaire distributed at the end of the semester, students generally reported that they found the clock project challenging and well organized. As we expected, the students indicated that building the clock kit was frustrating but most found the patent and manufacturing assignments very rewarding. In their comments, students observed that the course taught several valuable skills: how to follow instructions, write a concise description, and use language with precision. One student accurately but tersely revealed that the course simply taught her "Patience." Students also remarked that they learned to "think like an engineer" and that they learned for the first time that engineering involves more than math and science.

On the basis of class discussions and the take-home final exam, we observed that students absorbed several broader lessons. First, we could see that the students had broken down the barriers by which they frequently compartmentalize their education. By the end of the semester, they were freely drawing on skills and ideas acquired in their other courses to complete the patent and manufacturing assignments. To us, lowering these barriers is essential for engineering education since successful practitioners need to have exactly this sort of flexibility to solve new and expected problems.

Second, the students had learned that writing and speaking are not peripheral to engineering practice but indeed central to how engineers work. In their take-home exams, the more articulate students commented on different modes of representation and analysis in engineering (writing, drawing, calculating) and compared the strengths and weaknesses of each mode. Moreover, by looking at how students selectively used numbers and diagrams in the patent and manufacturing proposal, we could observe how they were learning how to choose representations and descriptions appropriate for both lay and professional audiences. Of particular interst is the necessary adaptations to students made to write a set of instructions for building the clock and a technically appropriate patent application. The constrast between these two assignments illustrated for the students the 
diversity of the audiences they will deal within their careers, and hopefully provided them with a bridge between their previous experiences with technology as consumers and their new experiences with technology as producers.

Although the students found it difficult to articulate, a third lesson of the course was the discovery by the students that they already possessed key skills necessary for success in engineering. As the clock project progressed, many students found that they drew on activities and experiences which they would not normally associate with studying science or engineering. In the questionnaires, students mentioned that they had built models or played with Legos while growing up. In doing so, they had already experienced the frustration of not getting something to work like it should. Equally, most had had dealt at some point with the problems of translating two-dimensional directions into three-dimensional artifacts while assembling ship models or readymade furniture. Significantly, we noticed that both male and female students reported having earlier relevant experiences with kits and instructions; while the women students were less likely to think of needlepoint or origami as being related to technology, these hobbies nonetheless taught them the importance of communicating through words and diagrams. Hence, through the clock project, students became aware of the skills they had acquired as the consumers of selected technologies, and an awareness of these skills may prove to be a valuable foundation as they study engineering and learn to be producers of new technology.

Overall, our experience with the clock project reveals the value of including a hands-on exercise in firstyear engineering courses. Through this project, our students honed their professional writing skills, and they did so because the assignments were directly related to the hands-on exercise. Moreover, they got a taste of different facets of engineering--including design, patenting, and manufacturing--and perhaps this taste convinced them that engineering can be an exciting and vital career. But most of all, by showing the students how engineers use different forms of representation to solve problems and communicate solutions, we gave the students a framework for thinking about and organizing their coursework. If the students find it useful to think about future courses in terms of expanding their repertoire of representational techniques, then the clock project is worth the time and effort.

\section{References}

1. Vincent Ercolano, "From Sleep 101 to Success 101.” ASEE Prism (September 1995), 24-29.

2. Donald Norman, Things that Make Us Smart: Defending Human Attributes in the Age of the Machine. Reading, Mass.: Addison-Wesley, 1993.

3. Eugene S. Ferguson, Engineering and the Mind's Eye. Cambridge: MIT Press, 1992.

4. Ryan D. Tweney, "Faraday's Notebooks: The Active Organization of a Creative Science," Physics Education 26:301-6 (September 1991).

5. Anne Eisenberg, Effective Technical Communication, 2 ed. New York: McGraw-Hill, 1992.

6. Thomas A. Edison, "Phonograph or Speaking Machine." U.S. Patent 200,521 (19 Feb. 1878)

7. Carlo M. Cipolla, Clocks and Culture, 1300-1700. New York: Norton, 1978.

8. David S. Landes, Revolution in Time: Clocks and the Making of the Modern World. Cambridge: Harvard University Press, 1983.

9. David Pressman, Patent It Yourself! How to Protect, Patent, and Market Your Inventions. New York: McGraw-Hill, 1979. 


\section{About the Authors}

W. BERNARD CARLSON is Associate Professor of Technology, Culture, and Communication in the School of Engineering and Applied Science at the University of Virginia. His specialty is the history of American technology and business. With Michael E. German, he is currently studying how Alexander Graham Bell and Thomas Edison employed sketches, notes, and models in developing the telephone in the 1870s.

KARIN PETERSON is completing her Ph.D in sociology at University of Virginia. Her research interests include the sociology of culture and knowledge. She was the teaching assistant in TCC 101 during the fall semester 1995. 


\section{Appendix -- Carlson -- TCC 101 -- Fail 1995 Schedule}

Monday

Week
Wednesday

Friday

1 September

Introduction to the course

In-class writing sample

4 September

What is engineering?

Discuss Hoover (handout)

Introduce Florman book

How to read a book

5

11 September

What is engineering?

History of Engineering

Read Florman, chs. 3-4

18 September

Work on clocks in class

Role of notebooks in engineering

What is engineering?

Read Florman Ch. 1

Assignment to find out

more about engineering fields

13 September

What is engineering?

Two page report and

brief group

presentation on

engineering fields

9

20 September

Read Eisenberg, 378-82

Florman essay due

11

25 September

Students demonstrate

clocks in class

Read Norman, Chs. 1-2

27 September

Cognition and Technology

Turn in notebooks

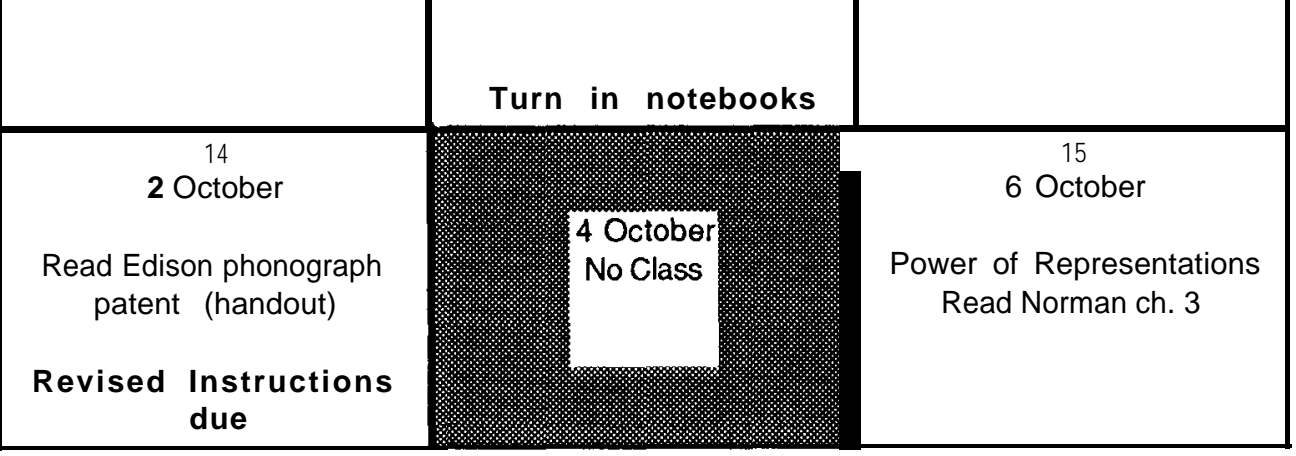

Time management and study skills, presented by

the Counseling Center

(tentative)

15 September

Introduce clock project

Introduce instruction

revision assignment

10

24 September

Writing instructions

Read Eisenberg, 174-84

Cognition and Technology

Read Norman, ch. 5

29 September

Read Norman, ch. 5

1996 ASEE Annual Conference Proceedings 


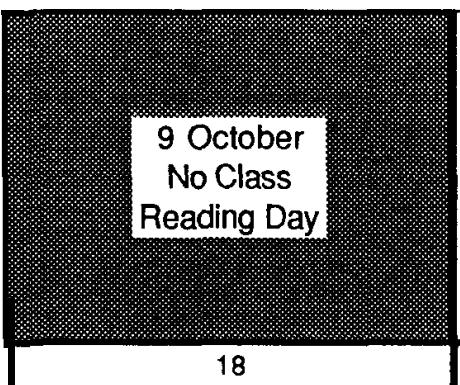

8

9

10
16 October

$11 \stackrel{16}{\text { October }}$

Introduce technical description assignment

Read Eisenberg, 147-66

Introduce patent assignment; organize groups

$\frac{\text { groups }}{23 \text { October }}$

What is a patent? History of patents

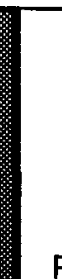 .}

180




\section{2 \\ 20 November \\ No class}

Students present roblem-solving strategy

to instructors in office

27 November

14

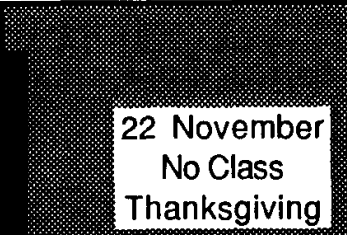

34

29 November

ead Florman, chs. 18-19

\section{November \\ No Class \\ Thanksgiving}

The Civilized Engineer
Read Florman, chs. 20-22
1 December

No Class

Proposals due 37

8 December

Group presentations of manufacturing plans

Conclusion and assessment

of course

Take-home final due during exam week 